OPEN ACCESS

Edited by:

Gianni Ciofani,

Politecnico di Torino, Italy

Reviewed by:

Gozde Ozaydin Ince,

Sabanci University, Turkey

Satoshi Arai,

Waseda University, Japan

Filippo Rossi,

Politecnico di Milano, Italy

*Correspondence:

Giulia Suarato

givlia.suarato@iit.it

Athanassia Athanassiou athanassia.athanassiou@iit.it

Specialty section:

This article was submitted to

Nanobiotechnology,

a section of the journal

Frontiers in Bioengineering and

Biotechnology

Received: 27 July 2018

Accepted: 13 September 2018

Published: 02 October 2018

Citation:

Suarato $G$, Bertorelli R and Athanassiou A (2018) Borrowing From

Nature: Biopolymers and

Biocomposites as Smart Wound Care

Materials.

Front. Bioeng. Biotechnol. 6:137.

doi: 10.3389/fbioe.2018.00137

\section{Borrowing From Nature: Biopolymers and Biocomposites as Smart Wound Care Materials}

\author{
Giulia Suarato $^{1,2 *}$, Rosalia Bertorelli ${ }^{2}$ and Athanassia Athanassiou ${ }^{1 *}$ \\ ${ }^{1}$ Smart Materials, Istituto Italiano di Tecnologia, Genoa, Italy, ${ }^{2}$ In vivo Pharmacology Facility, Istituto Italiano di Tecnologia, \\ Genoa, Italy
}

Wound repair is a complex and tightly regulated physiological process, involving the activation of various cell types throughout each subsequent step (homeostasis, inflammation, proliferation, and tissue remodeling). Any impairment within the correct sequence of the healing events could lead to chronic wounds, with potential effects on the patience quality of life, and consequent fallouts on the wound care management. Nature itself can be of inspiration for the development of fully biodegradable materials, presenting enhanced bioactive potentialities, and sustainability. Naturally-derived biopolymers are nowadays considered smart materials. They provide a versatile and tunable platform to design the appropriate extracellular matrix able to support tissue regeneration, while contrasting the onset of adverse events. In the past decades, fabrication of bioactive materials based on natural polymers, either of protein derivation or polysaccharide-based, has been extensively exploited to tackle wound-healing related problematics. However, in today's World the exclusive use of such materials is becoming an urgent challenge, to meet the demand of environmentally sustainable technologies to support our future needs, including applications in the fields of healthcare and wound management. In the following, we will briefly introduce the main physico-chemical and biological properties of some protein-based biopolymers and some naturally-derived polysaccharides. Moreover, we will present some of the recent technological processing and green fabrication approaches of novel composite materials based on these biopolymers, with particular attention on their applications in the skin tissue repair field. Lastly, we will highlight promising future perspectives for the development of a new generation of environmentally-friendly, naturally-derived, smart wound dressings.

Keywords: wound healing, biomimetic, alginate, chitosan, hyaluronic acid, silk fibroin, keratin, antibacterial

\section{INTRODUCTION}

Skin is our major external defense system, in charge of protecting our inner body structures from microorganisms' attacks, and the adverse effects of the external environment. Adult skin is composed of three layers: epidermis or stratum corneum, mainly consisting of keratinocytes; dermis, the connective tissue rich in collagen; and hypodermis or subcutaneous layer, composed of fat tissue, which provides thermal isolation and mechanical protection to the body (Gurtner et al., 2008). 
Wounds are breaks or defects within the skin, which may form due to physicochemical or thermal damage. Acute wounds define injured tissues that need a healing period over 8-12 weeks, (e.g., burns, chemical injuries, cuts). In contrast, chronic wounds are a fallout of diseases, such as venous or arterial vascular insufficiency, pressure necrosis, cancer, and diabetes (Sen et al., 2009; Moura et al., 2013). They require longer healing time (weeks-months to years) and often fail to reach a normal healthy state, persisting in a pathological condition of inflammation (Guo and Dipietro, 2010). Therefore, delayed or impaired wound healing poses a significant socio-economic burden on patients and health care systems worldwide, in terms of treatment costs and waste production (Sen et al., 2009).

Insight into the intricate biochemical events activated during skin repair is crucial to design appropriate wound dressings (Weller and Sussman, 2006; Gurtner et al., 2008; Pereira et al., 2013). The healing process can be divided into the following, overlapping stages: homeostasis, inflammation, proliferation, and remodeling (Martin, 1997; Gurtner et al., 2008; Bielefeld et al., 2013; Das and Baker, 2016). Homeostasis is the immediate response of the body to an injury, in order to stop blood loss at the wound site, by means of fibrin cloths as temporary barriers (Sinno and Prakash, 2013). Inflammation (form $24 \mathrm{~h}$ to 4-6 days) is mediated by neutrophils and macrophages (Broughton et al., 2006), that sweep the wound bed from foreign particles and tissue debris. Cytokines and enzymes are released to stimulate fibroblasts and myofibroblasts (Das and Baker, 2016), while the wound exudate provides the essential moisture for the recovery. In the proliferation phase epithelialization occurs and newly formed granulation tissue begins to fill the wound area, producing new extracellular matrix (ECM). Finally, during the remodeling phase, collagen-based cross-linking is responsible for a tight 3D network formation, increasing the tensile strength of the new tissue (Sinno and Prakash, 2013).

Given the multiple mechanisms involved in the skin wound healing and the interplay of several external factors, the choice of suitable dressing materials is compelling. Specifically, for biodegradable natural materials, their degradation needs to follow the dynamics of the wound repair, guaranteeing the physiological healing evolution, and releasing active principles when needed. At last but not least, proper consideration should be put onto the environmental sustainability of these biomaterials, in terms of green chemistry fabrication approaches, and complete biodegradation without harmful by-products. While numerous reviews on traditional wound dressing biomaterials have been extensively published (Sell et al., 2010; Mogoşanu and Grumezescu, 2014; Norouzi et al., 2015; Mele, 2016), in this mini-review we will focus our attention on the most recent naturally-derived, active systems, pursuing the quest for an environmentally sustainable wound management.

\section{MIMICKING NATURE AS A THERAPEUTIC STRATEGY}

Successful wound management relies on understanding the healing process combined with a know-how on the properties of the various dressing materials available. Principal purpose of any wound treatment is to maximize the treatment efficiency (Weller and Sussman, 2006). Currently, standard care procedures consist of swabbing the infection, cleaning the wound bed from tissue debris, and applying the dressing (Dreifke et al., 2015). In case of extended skin lesions, the use of split-thickness skin autografts or allografts might be required, carrying safety issues related to disease transmission and immune rejection.

An ideal dressing should remove excessive exudate to avoid tissue maceration and promote autolytic debridement, while keeping moisture, adequate oxygen and water vapor permeability within the wound. It should be adhesive and flexible, to favor mechanical compliance to the patient body and ease the application/removal. Deliverable bioactive compounds, such as antibiotics, essential oils, and natural antioxidants, stimulate the dressing interaction with the wound microenvironment and further enhance the therapeutic action via antimicrobial, antifungal, and antiseptic activities (Pereira et al., 2013). A number of fabrication techniques, such as film-casting, electrospinning, self-assembly, freeze-drying, emulsions, microsphere injection, have been employed to produce wound dressings, either based on synthetic macromolecules or on materials of natural origin (Sell et al., 2007, 2010; Wei and Ma, 2008; Huang and Fu, 2010; Zhong et al., 2010; Rieger et al., 2013; Mogoşanu and Grumezescu, 2014; Norouzi et al., 2015; Liakos et al., 2016; Mele, 2016).

Recently, natural biopolymers have largely attracted the scientific community interest. On top of their notable biocompatibility and biodegradability, natural occurring proteins and polysaccharides allow to achieve the highest level of biomimicry, recapitulating the native ECM biological and physico-chemical features. Further architectural resemblance can be obtained with an appropriate processing (e.g., nanofibers, sponge-like hydrogels; Huang and Fu, 2010; Mogoşanu and Grumezescu, 2014; Liakos et al., 2015; Mele, 2016). Despite their batch-to-batch variations in terms of mechanical properties and their rather limited shelf-life, naturally-derived biopolymers confer ECM support (collagen, gelatin, hyaluronic acid), present cell-recognition domains and biomolecule binding sites (RGD and LDV sequences in silk fibroin and keratin), and may possess inherent antibacterial and anti-inflammatory properties (chitosan, alginate). Moreover, in the past decades, clinical understanding advancements have directed significant exploitation of natural materials in clinical trials (Vyas and Vasconez, 2014; Dhivya et al., 2015). By looking into our natural surroundings and by re-using some of the discarded natural resources, several functional biomaterials can be easily identified and implemented for promising wound healing applications, with a reduced impact on the environment (Figure 1).

\section{PROTEIN-BASED BIOPOLYMERS Collagen and Gelatin}

Collagen is the most abundant animal protein, which provides mechanical strength to tissues and stimulates cell-adhesion and proliferation (Neel et al., 2013; An et al., 2016). Twentynine different types of collagen have been identified, displaying a triple-helical tertiary structure of polypeptide sequences (Figure 2a), but only a few are used in the production of 


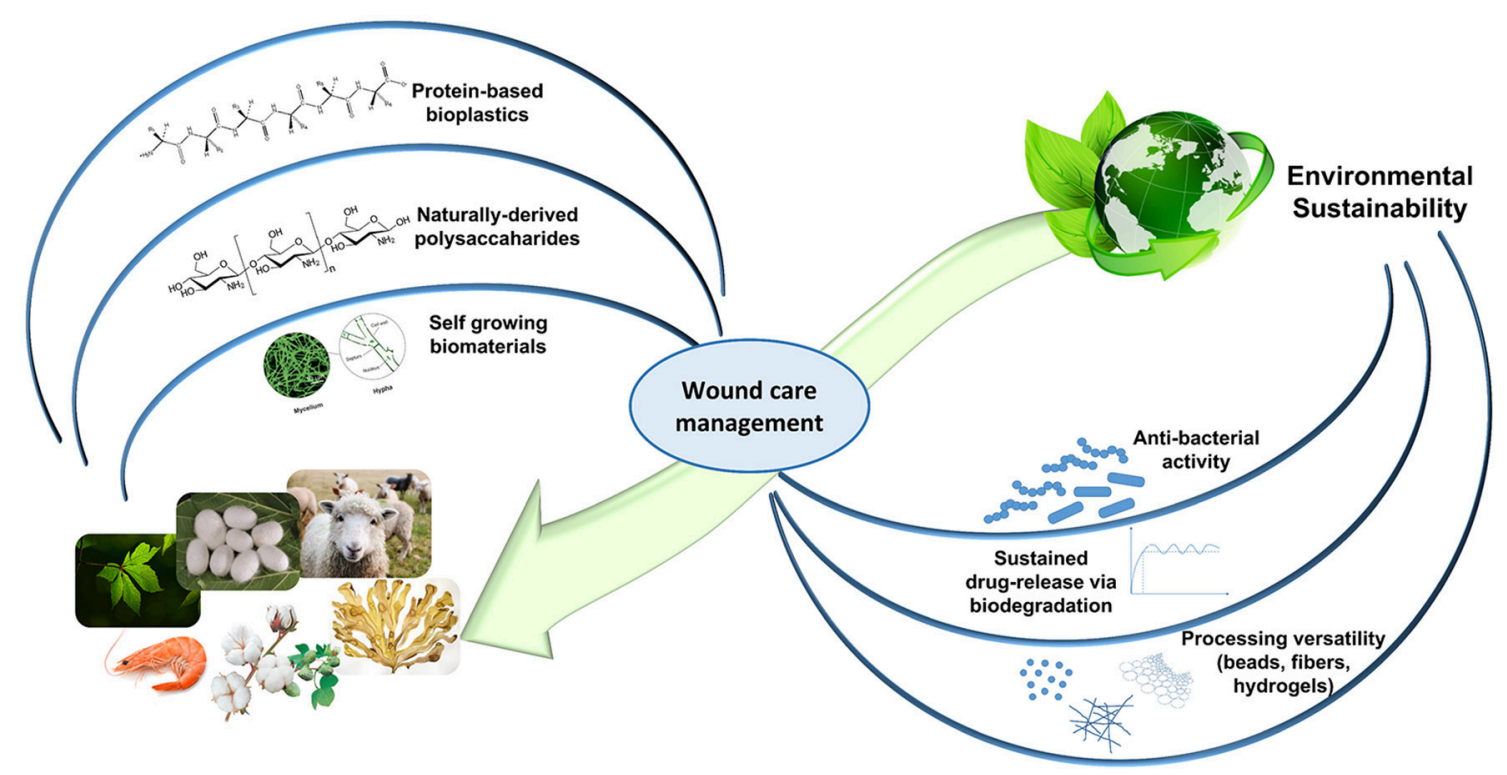

FIGURE 1 | The circularity concept of Nature-mimicking for an environmentally-friendly wound healthcare. Self-growing biomaterials panel is adapted from Haneef et al. (2017). This material is licensed under the Creative Commons Attribution 4.0 International Public License (https://creativecommons.org/licenses/by/4.0/ legalcode).

collagen-based biomaterials. As animal-derived proteins may be responsible for allergic reactions and pathogen transmissions (Koide, 2007), an alternative is constituted by collagen from heterologous expression in mammalian, insect and yeast cells (Olsen et al., 2003), or produced by Escherichia coli (Pinkas et al., 2011). High biocompatibility and biodegradability by endogenous collagenases make collagen ideal for biomedical applications (Parenteau-Bareil et al., 2010; Chattopadhyay and Raines, 2014). During wound healing, fibroblasts produce collagen molecules that aggregate to form fibrils with diameter in the range of $10-500 \mathrm{~nm}$. This fibrous network facilitates cell migration to the wounded site, actively supporting tissue repair (Baum and Arpey, 2005).

Thanks to a facile chemical functionalization of the protein structure, various dressing architectures have been exploited. Collagen-based wound dressings, either in forms of hydrogels, electrospun fibers, or nanocrystal-containing scaffolds, have been applied to cover burn wounds, treat ulcers (Ghica et al., 2017; Guo et al., 2017; Bhowmick et al., 2018; Yoon et al., 2018), reduce tissue contraction and scarring, and increase epithelialization rate (Powell et al., 2008). Collagen sponges and fibrous membranes were found particularly promising, due to their wet-strength that allows suturing to soft tissues and provides a template for new tissue growth. Composites with other natural materials, such as dextran, chitosan, hyaluronic acid, and alginate (Karri et al., 2016; Ghica et al., 2017; Wei et al., 2018) or constructs based on collagen and synthetic biopolymers, such as poly- $\alpha$-hydroxyl esters (Hall Barrientos et al., 2017; Albright et al., 2018; Bhowmick et al., 2018) have been extensively exploited. Moreover, acetylated, succinylated, methylated, or biotinylathed collagen have been used to immobilize therapeutic enzymes or growth factors and to control drug delivery (Lima et al., 2015; Mele, 2016; Qu et al., 2018; Zhu et al., 2018). Albright and coworkers (Albright et al., 2018) proposed a multi-structured nanofibrous dressing, composed of poly- $\varepsilon$-caprolactone/collagen electrospun matrix, loaded with transforming growth factor TGF- $\beta 1$ and modified with polypeptide-based nanocarriers incorporating tannic acid and gentamicin. The multifunctional platform showed anti-bacterial and anti-inflammatory properties, while retaining a favorable topography for cell proliferation, thus accelerating healing and wound closure. A similar construct was proposed by Karri et al. (2016), where a composite scaffold of collagen and alginate was impregnated with curcuminloaded chitosan nanoparticles to obtain an all-natural wound dressing.

A collagen-derivative with promising biomedical values is gelatin. Gelatin is obtained by an incomplete denaturalization of collagen extracted from connective tissues, skin, and boiling bones (Jaipan et al., 2017). It has been employed to fabricate strong hydrogel-like membranes (Thanusha et al., 2018), microspheres (Thyagarajan et al., 2017), sponges, and electrospun mats (Chen et al., 2016), for dermal tissue applications and to treat severe burn wounds. Various combinations of gelatin and modified chitosan have been proposed (Han et al., 2014; Agarwal et al., 2016), as well as blends with poly-vinyl alcohol based via enzymatic crosslinking, to support fibroblast culture and proliferation (Hago and Li, 2013).

Despite their rather extensive usage as biomaterials for scaffold design, collagen and gelatin remain sustainable materials with highly engineering potential yet unexplored (Hall Barrientos et al., 2017; Golser et al., 2018). 


\section{a Protein-based Biopolymers}

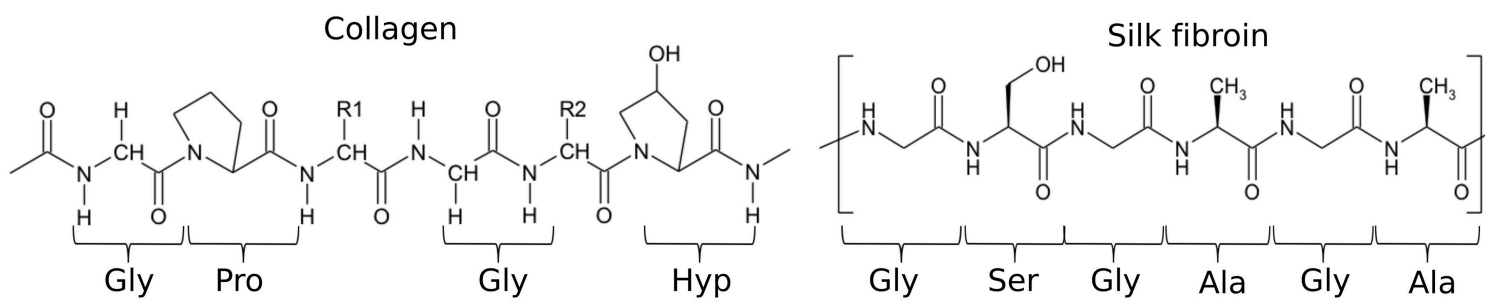

b Natural Polysaccharides

Hyaluronic Acid

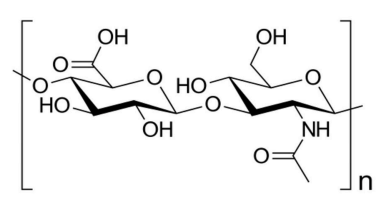

\section{Chitosan}

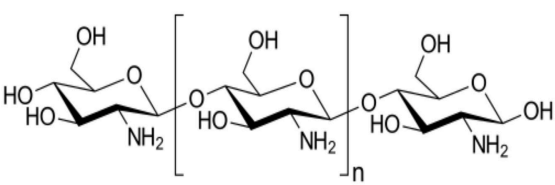

Alginate

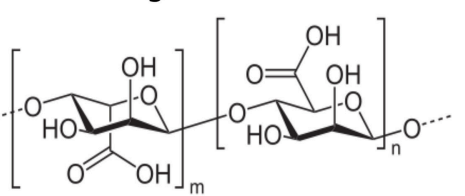

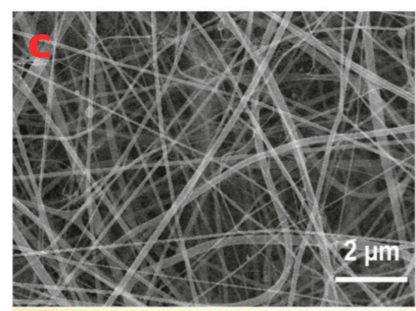
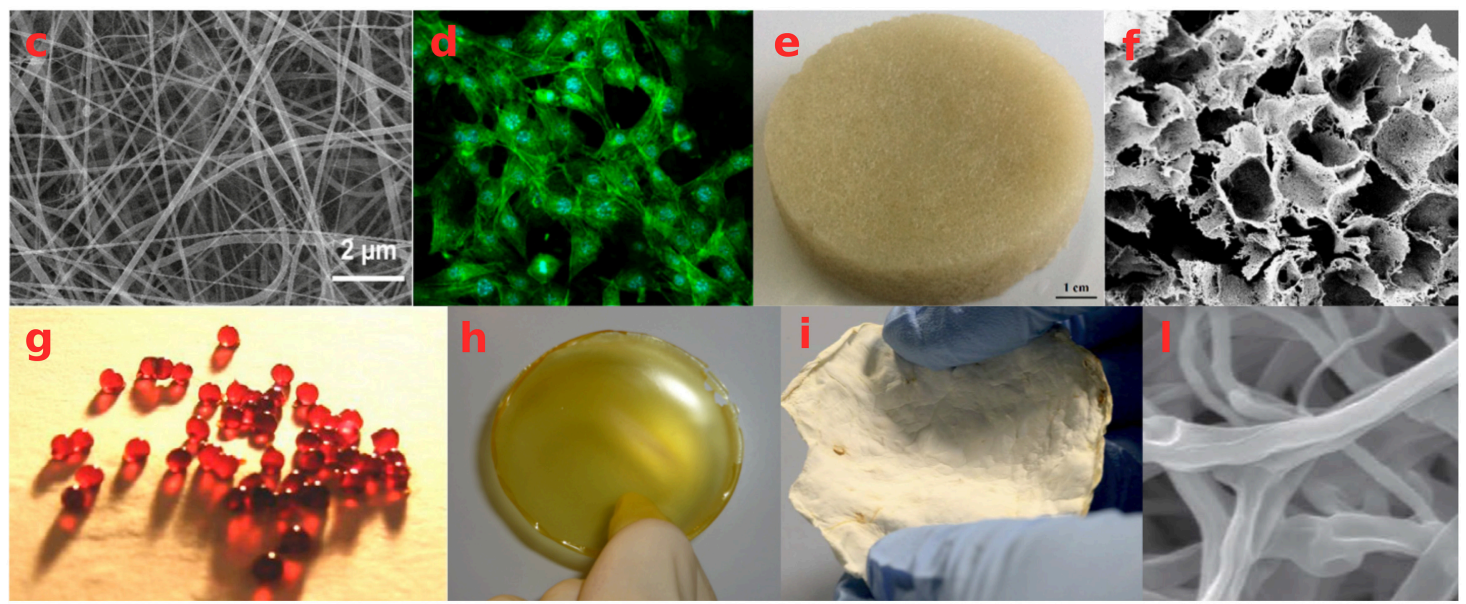

FIGURE 2 | Naturally-derived biopolymer-based structures with potential application as wound healing systems. (a) examples of protein-based biopolymers primary structures-aminoacidic sequence of collagen type I molecules and aminoacidic sequence of silk fibroin molecules: Gly, glycine; Ala, alanine; Pro, proline; Ser, serine; Hyp, hydroxyproline; (b) natural polysaccharide structures - hyaluronic acid, chitosan, and alginate; (c,d) Biocompatible silk/parsley electrospun fibers (average

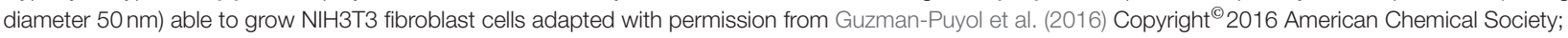
$\mathbf{( e , f )}$ wool keratin sponges, reprinted from Patrucco et al. (2016) Copyright ${ }^{(} 2016$ with permission from Elsevier; (g) calcium cross-linked alginate beads and (h) film incorporating antiseptic PVPI complex, reprinted from Liakos et al. (2013) Copyright ${ }^{\circledR} 2013$ with permission from Elsevier; (i,I) mycelia material from P. ostreatus after 20 days of growth on potato-dextrose broth and cellulose, presenting a 3D network of hyphae. Panels (i,I) are adapted from Haneef et al. (2017). This material is licensed under the Creative Commons Attribution 4.0 International Public License (https://creativecommons.org/licenses/by/4.0/legalcode).

\section{Silk Fibroin}

Silks are proteins produced in the epithelial cells of specialized glands of various arthropods, such as spiders and silkworms. The secreted silk fibers present a highly repetitive sequence, consisting mainly of glycine (43\%), alanine (30\%), and serine $(12 \%)-[G A G S G A]_{n}$ motifs, arranged in $\beta$-sheets regions embedded in an amorphous matrix (Chutipakdeevong et al., 2013; Reimers et al., 2015), which confer high toughness and elasticity (Figure 2a). Regarding more specifically silk from the cocoons of Bombyx mori silkworms, two kinds of proteins are its major components: the fibroin and the sericin. The fibroins are composed of three types of protein fibers: $350 \mathrm{kDa}-$ heavy chain, $30 \mathrm{kDa}$-glycoprotein, and $25 \mathrm{kDa}$-light chain, the latter conferring hydrophilicity, water uptake ability and cell adhesion properties. Light and heavy chains are connected by disulfide bonds, while the glue protein sericin coats the silk fibers. Thanks to high mechanical resistance, enzymatic-driven biodegradability and favorable cell attachment, silk fibroins have been successfully exploited for skin tissue engineering and wound healing applications.

Bombyx mori silkworm-derived fibroins are obtained from cocoon and separated from sericin by degumming in alkaline boiling water and following solubilization in hot $\mathrm{LiBr}$ solution (Reimers et al., 2015). Regenerated silk water soluble form, silk I, can be converted into insoluble silk II, by modifying the $\alpha$-helical chain arrangements into $\beta$-sheets via alcohol treatment 
or water vapor annealing (WVA; Min et al., 2006; Wharram et al., 2010; Hu et al., 2011). In fact, by controlling the protein secondary structure, fibroin scaffolds' biodegradation can be properly tuned, in order to modulate the release of bioactive molecules (Hofmann et al., 2006), such as antibiotics (Pritchard et al., 2013; Chouhan et al., 2017), growth factors (Schneider et al., 2009; Chouhan et al., 2017; Pignatelli et al., 2018), and anti-oxidant compounds (Fan et al., 2012; Sheng et al., 2013; Lin et al., 2016). In their study, Pignatelli and coworkers (Pignatelli et al., 2018) encapsulated human platelet lysate into electrospun silk-PEO patches, to prolong the growth factor shelf life and ease its handling during wound management. By changing the crystallinity degree of the fibrous matrices via WVA from 21 to 35 or $44 \%$, the 24 -h drug release drastically decreased from 100 to 80 and $46 \%$, respectively.

Proteins' versatile nature allows for a plethora of processing techniques, with the consequent fabrication of multiple scaffold morphologies, such as films (Srivastava et al., 2015), foams and sponges (Roh et al., 2006), gels, and fibrous matrices. In the last decade, electrospun fibroin has been extensively proposed for the design of anti-bacterial, anti-inflammatory and anti-oxidant patches (Lin et al., 2016; Selvaraj and Fathima, 2017; Yang et al., 2017). To ease a water-based electrospinning process, silk has been processed either in combination with natural polymers, such as cellulose (Guzman-Puyol et al., 2016; Figures 2c,d), gelatin (Shan et al., 2015), sericin (Hang et al., 2012), chitosan (Cai et al., 2010), alginate (Roh et al., 2006), elastin (Zhu et al., 2016), and hyaluronic acid (Yan et al., 2013), or mixed with synthetic materials, such as polyethylene oxide (Schneider et al., 2009; Wharram et al., 2010; Chutipakdeevong et al., 2013), polyvinyl alcohol (PVA; Chouhan et al., 2017), and polyhydroxy esters (Lian et al., 2014; Shahverdi et al., 2014; Suganya et al., 2014; Shanmugam and Sundaramoorthy, 2015). Silk/PVA mats loaded with Ciprofloxacin and epidermal growth factors (Chouhan et al., 2017) enhanced human dermal fibroblasts and keratinocytes proliferation in vitro, and favored re-epithelization, mature collagen deposition and complete wound closure at 14 days in a in vivo wound healing rabbit model. In a different work, Ju and coworkers (Ju et al., 2016) investigated the intrinsic anti-inflammatory effects of a porous fibroin/PEO electrospun nanomatrix in a mice burn-model, observing downregulation of pro-inflammatory cytokines IL- $1 \alpha$ and IL-6.

\section{Keratin}

Keratins (Ker) are the most abundant group of insoluble and filament-forming proteins produced in epithelial cells of mammals, birds, reptiles, and humans. As structural components of wool, nails, horn, feathers, and hair, they exploit mechanical support and protective functions against the environment (Reichl, 2009; Wang et al., 2016). Keratins present a complex intermediate filament (IF)-matrix hierarchical structure and are categorized according to the polypeptide chain secondary assembly. $\alpha$-Ker $(40-68 \mathrm{kDa})$ comprise $\alpha$-helices arranged in coiled-coil heterodimers to form 7-nm IF, while $\beta$-Ker (10-22 $\mathrm{kDa})$ consist of packed $\beta$-sheets disposed in 3-nm IF. The highsulfur containing matrix ( $\gamma$-Ker, below $10 \mathrm{kDa})$, rich in cysteine, tyrosine, glycine and phenylalanine residues, present a globular assembly (Dowling et al., 1986; Fraser et al., 1986; Steinert and Marekov, 1993; Rouse and Van Dyke, 2010; Wang et al., 2016). The secondary structure of keratinous materials largely affects their mechanical resistance, solubility, and hydration sensitivity (Wang et al., 2016). Tons of Ker-containing biomasses are produced every year, from meat and poultry market, wool industry and hair salons, leading to continuous accumulation of wastes in the ecosystem. The challenges associated with this waste disposal have been considered by the European Parliament and Council regulation EC 1774/2002 (Sharma and Gupta, 2016). Due to the presence of strong disulfide and H-bonds, keratin extraction from biomasses involves rather complicated methods, such as microbial and enzymatic hydrolysis, mechanical treatments, or chemical protocols with alkali, reducing agents or ionic liquids (Yamauchi et al., 2003; Ozaki et al., 2014; Sharma and Gupta, 2016; Shavandi et al., 2017).

However, thanks to its biocompatibility, biodegradability, and hemostatic properties, keratin constitutes a potential green secondary raw material for wound healing, tissue repair, drug delivery, and cosmetics applications (Sharma and Gupta, 2016; Arslan et al., 2017; Shavandi et al., 2017). Since its earliest documented use in medicinal applications (China, sixteenth century; Rouse and Van Dyke, 2010), in the past decades several Ker-based biomaterials have been proposed, given the ability of this biopolymer to self-assembly into 3D networks favorable for cell infiltration, and its intrinsic bioactivity for the presence of cell binding motifs (such as EDS, LDV, and RGD; Rouse and Van Dyke, 2010). Keratin extracted from chicken feathers, wool and human hair have been processed in films (Yamauchi et al., 2003; Fujii and Ide, 2004; Tonin et al., 2007; Reichl, 2009; Cui et al., 2013), sponge-like and hydrogel-like scaffolds for tissue engineering and wound healing (Figures 2e,f; Tachibana et al., 2002; Verma et al., 2008; Hill et al., 2010; Saul et al., 2011; Richter et al., 2012; Wang et al., 2012; Xu et al., 2013; Patrucco et al., 2016; Singaravelu et al., 2016). Electrospun fibers have also been obtained in combination with PEO (Aluigi et al., 2007, 2008; Fan et al., 2016; Ma et al., 2017), PVA (Choi et al., 2015; He et al., 2017; Wang et al., 2017), fibroin (Zoccola et al., 2008; Yen et al., 2016), poly-caprolactone (Boakye et al., 2015; Edwards et al., 2015; Li et al., 2016; Zhu et al., 2017), poly(3-hydroxybutyrate-co-3-hydroxyvalerate) (Yuan et al., 2015), chitosan (Singaravelu et al., 2016), and gelatin (Yao et al., 2017).

\section{NATURALLY-DERIVED POLYSACCHARIDES}

\section{Hyaluronic Acid}

Hyaluronic acid (HA) is a non-immunogenic polysaccharide consisting of glucuronic acid and N-acetyl-D-glucosamine units (Figure 2b). This glycosaminoglycan is one of the main components of the connective tissue in mammals (Mele, 2016). Due to its hygroscopic nature, HA has been used to prepare hydrogel-like constructs, to support keratinocyte migration and angiogenesis, and promote a scar-free wound healing (Mogoşanu and Grumezescu, 2014; Dreifke et al., 2015). The molecular 
weight (MW) plays a key role in the process (Tolg et al., 2014): low MWHA degradation products were found to be proinflammatory (Campo et al., 2010; Dreifke et al., 2015), while high MWHA appeared to inhibit nutrient supply. Interestingly, medium MWHA (100-300 kDa) showed enhanced wound closure capability through up-regulation of adhesion molecules (Ghazi et al., 2012). Moreover, the hydrophilicity of the HA chains allows the 3D network swelling and the consequent gradual release of encapsulated active compounds, making this biomaterial suitable as drug delivery platform (Maeda et al., 2014). HA-based electrospun fibers, either pure or in combination with other biomacromolecules (Xu et al., 2009; Hsu et al., 2010; Uppal et al., 2011; Dogan et al., 2016), have been proposed for tunable degradation and sustained release in vitro and in vivo.

\section{Chitosan}

Chitosan (CS), a deacetylated chitin-derivative found in the exoskeletons and shells of crustaceans, is a linear polysaccharide consisting of $\beta(1-4)$-D-glucosamine and $\mathrm{N}$-acetyl-D-glucosamine groups randomly distributed (Figure 2b). Owing to its intrinsic antifungal, antibacterial, hemostatic, and muco-adhesive properties, chitosan has been widely exploited in the biomedical field for wound and burn treatments (Dash et al., 2011; Croisier and Jérôme, 2013; Norouzi et al., 2015; Zhao et al., 2015). Several dressing architectures have been proposed: CS-Aloe vera membranes (Wani et al., 2010), thyme oil-CS films (Altiok et al., 2010), CS-gelatin sponges (He et al., 2007), CS-silk hydrogels (Silva et al., 2012), CS-cellulose films (Niyas Ahamed and Sastry, 2011; Romano et al., 2015a), cinnamon oil-CS/polyethylene oxide nanofibers (Rieger and Schiffman, 2014), and CS/poly(3-hydroxybutyrateco-3-hydroxyvalerate) scaffolds (Veleirinho et al., 2012). In addition, water-soluble derivatives, such as carboxymethylCS and methacrylate glycol CS have been synthetized and investigated for wound healing applications (Romano et al., 2015b).

\section{Alginate}

Alginate $(\mathrm{Alg})$ is a linear co-polymer of $\beta$-D-Mannuronic acid and $\alpha$-L-Glucuronic acid (Figure $2 \mathbf{b}$ ). This polysaccharide is mostly abundant in Brown Algae or produced by some bacteria (Khan and Ahmad, 2013). It is highly hydrophilic, biocompatible, and able to absorb wound exudate, maintaining a moist microenvironment (Chiu et al., 2008). The combination of alginate with antimicrobial and enzymatic components can promote elimination of necrotic tissues and microbial bodies, while the polysaccharide base can stimulate reparative wound processes (Patel et al., 2007). Alginate dressings are also useful as delivery platforms, in order to provide a controlled release of therapeutic substances to exuding wounds (e.g., pain-relieving, antibacterial, and anti-inflammatory agents; Maver et al., 2015; Szekalska et al., 2016; Setti et al., 2018). Biodegradable NaAlg/PVPI (povidone iodine complex) films and Ca-Alg/PVPI beads have displayed antimicrobial and antifungal activities (Liakos et al., 2013; Figures 2g,h). Moreover, Na-Alg/PVPI films have shown to reduce the inflammatory response and accelerate the wound healing providing a controlled release of PVPI (Summa et al., 2018). To treat UV-induced skin burns, instead, electrospun nanofibers loaded with lavender essential oil have been used: the composite mats exhibited antibacterial and antiinflammatory properties, being able to reduce the production of pro-inflammatory cytokines both in vitro and in vivo (Hajiali et al., 2016).

\section{SELF-GROWING MYCELIUM-BASED BIOMATERIALS}

Mycelium, the fungi vegetative part, comprises a network of filamentous hyphae, which penetrate the substrate. Hyphae are tubular structures of micrometric diameter, composed of aligned and elongated cells, separated by walls, called septa (Figures 2i,1). A continuous cell wall protects the hyphae and confers mechanical strength and shape to the mycelium (Haneef et al., 2017; Jones et al., 2017). Being constituted of chitin, chitosan, glucans, mannoproteins, and glycoproteins (Synytsya and Novák, 2013), the cell wall is a biopolymer composite that prevents the hyphae from collapsing during their sprouting and movement (Cairney, 2005).

Peculiarity of these living, self-growing composites is the possibility to tune the physico-chemical properties during their growth phase, reducing sophisticated processing and byproduct formation, while allowing for ready-to-use systems. Throughout its dynamic growth, the mycelium "senses" the substrate and responds to the surrounding, depending on edaphic conditions, substrate $\mathrm{pH}$ and composition, or the presence of other living organisms (Krull et al., 2013). A polarized extension of the cell wall occurs at the apical region of the hyphae, as the mycelium secretes a variety of enzymes, hydrolyzes the substrate and absorbs the solubilized nutrients. By properly exploiting different feeding substrates for hyphae digestion, the resulting properties of the interwoven fibrous mycelium material can be efficiently tailored. Type and amount of absorbed nutrients may affect mycelia growth rate, extension and biological activity (Frimpong-Manso et al., 2011; Da Silva et al., 2012; Larsen, 2015; Anderson and Cairney, 2018). Similarly, culture conditions and feeding substrate highly influence the final chemical composition (Krull et al., 2013; Haneef et al., 2017), either stimulating plasticizer biosynthesis (lipids and small glycoproteins) or promoting rigid macromolecule production (chitin, $\beta$-D-glucans; Synytsya and Novák, 2013). In this regard, mycelia can be considered as $3 \mathrm{D}$ smart, micro-reactors, able to bio-convert various agro-residues into enzymes, polysaccharides, and bioactive metabolites (Vassilev et al., 1995; Krull et al., 2013; Vamanu, 2014; Yan et al., 2014; Salati et al., 2017), with potential bio-pharmaceutical and neutraceutical relevance.

In the past, mycelia-derived scaffolds have been exploited ( $\mathrm{Su}$ et al., 1999, 2005; Hung et al., 2001). Su et al. (1997) developed a filament-structured membrane from the residue of Ganoderma tsugae, called Sacchachitin, composed of $\beta$-1,3-glucan (60\%), and $\mathrm{N}$-acetylglucosamine $(40 \%)$, to be used as skin substitute. The new biomaterial demonstrated wound healing potential in vivo, by promoting fibroblast proliferation and migration. In a 
following study (Su et al., 2005), the Sacchachitin membranes appeared to boost keratinocytes proliferation and prevent metalloproteinase-related ECM degradation, contributing to accelerate the healing process of a chronic wound in vivo model. Furthermore, a micronized Sacchachitin nanogel has been investigated to treat superficial chemical corneal burns in vivo (Chen et al., 2012), while the anti-oxidant and immunomodulating effects of extracts from mycelia of some medicinal fungi have been investigated for skin aging (Kim et al., 2014), dermatitis (Hwang et al., 2012), and UV-protection (Nanbu et al., 2011; Bae et al., 2012), suggesting a promising mycelia biological value yet unexplored.

\section{CONCLUSIONS - A QUEST FOR AN ENVIRONMENTALLY SUSTAINABLE WOUND MANAGEMENT}

Environmental sustainability has nowadays become an imperative issue to front, in an effort to balance both the industrial productivity and the planet ability to generate resources, with the neutralization of wastes and the mitigation of polluting processes. Material and energy consumption related to the healthcare industry, ranging from complex material manufacturing processes, to drug packaging, to high-volume

\section{REFERENCES}

Agarwal, T., Narayan, R., Maji, S., Behera, S., Kulanthaivel, S., Maiti, T. K., et al. (2016). Gelatin/Carboxymethyl chitosan based scaffolds for dermal tissue engineering applications. Int. J. Biol. Macromol. 93, 1499-1506. doi: 10.1016/j.ijbiomac.2016.04.028

Albright, V., Xu, M., Palanisamy, A., Cheng, J., Stack, M., Zhang, B., et al. (2018). Micelle-coated, hierarchically structured nanofibers with dual-release capability for accelerated wound healing and infection control. Adv. Healthc. Mater. 7:e1800132. doi: 10.1002/adhm.201800132

Altiok, D., Altiok, E., and Tihminlioglu, F. (2010). Physical, antibacterial and antioxidant properties of chitosan films incorporated with thyme oil for potential wound healing applications. J. Mater. Sci. Mater. Med. 21, 2227-2236. doi: $10.1007 / \mathrm{s} 10856-010-4065-\mathrm{x}$

Aluigi, A., Varesano, A., Montarsolo, A., Vineis, C., Ferrero, F., Mazzuchetti, G., et al. (2007). Electrospinning of keratin/poly(ethylene oxide) blend nanofibers. J. Appl. Polym. Sci. 104, 863-870. doi: 10.1002/app.25623

Aluigi, A., Vineis, C., Varesano, A., Mazzuchetti, G., Ferrero, F., and Tonin, C. (2008). Structure and properties of keratin/PEO blend nanofibres. Eur. Polym. J. 44, 2465-2475. doi: 10.1016/j.eurpolymj.2008.06.004

An, B., Lin, Y. S., and Brodsky, B. (2016). Collagen interactions : drug design and delivery. Adv. Drug Deliv. Rev. 97, 69-84. doi: 10.1016/j.addr.2015.11.013

Anderson, I. C., and Cairney, J. W. (2018). Ectomycorrhizal fungi: exploring the mycelial frontier. FEMS Microbiol. Rev. 31, 388-406. doi: 10.1111/j.1574-6976.2007.00073.x

Arslan, Y. E.,Sezgin Arslan, T., Derkus, B., Emregul, E., and Emregul, K. C. (2017). Fabrication of human hair keratin/jellyfish collagen/eggshellderived hydroxyapatite osteoinductive biocomposite scaffolds for bone tissue engineering : from waste to regenerative medicine products. Colloids Surf. B Biointerf. 154, 160-170. doi: 10.1016/j.colsurfb.2017. 03.034

Bae, J. T., Ko, H. J., Kim, G. B., Pyo, H. B., and Lee, G. S. (2012). Protective effects of fermented citrus unshiu peel extract against ultraviolet-a-induced photoageing in human dermal fibrobolasts. Phytother. Res. 26, 1851-1856. doi: $10.1002 /$ ptr.4670 medical wastes, might heavily contribute to increase the overall pollution, with an unsought negative impact on the human health (Jameton and Pierce, 2001). Nature itself can be of inspiration to develop cost-competitive, low-energy consumption and fully biodegradable materials, presenting greater environmental sustainability. The increasing interest of the scientific community in the use of either protein-based or polysaccharide-derived dressings is striking, and it reflects the growing perspective of giving back what we borrowed from Nature. In this regard, self-growing mycelia, which are biocomposites constituted of both proteins and polysaccharides, can represent a smart strategy to fabricate healthcare products of the future, as economically and environmentally valid alternatives to synthetic materials. In conclusion, naturally derived biopolymers provide a versatile, multifunctional, and tunable platform to design appropriate extracellular environments, able to actively contrast the onset of infections and inflammations, while promoting tissue regeneration, and scar remodeling.

\section{AUTHOR CONTRIBUTIONS}

GS and RB conceived and wrote the main manuscript text. AA edited and reviewed the whole manuscript.

Baum, C. L., and Arpey, C. J. (2005). Normal cutaneous wound healing: clinical correlation with cellular and molecular events. Dermatol. Surg. 31, 674-686. doi: 10.1097/00042728-200506000-00011

Bhowmick, S., Thanusha, A. V., Kumar, A., Scharnweber, D., Rother, S., and Koul, V. (2018). Nanofibrous artificial skin substitute composed of mPEG-PCL grafted gelatin/hyaluronan/chondroitin sulfate/sericin for 2nd degree burn care: in vitro and in vivo study. RSC Adv. 8, 16420-16432. doi: 10.1039/C8RA01489B

Bielefeld, K. A., Amini-Nik, S., and Alman, B. A. (2013). Cutaneous wound healing: recruiting developmental pathways for regeneration. Cell. Mol. Life Sci. 70, 2059-2081. doi: 10.1007/s00018-012-1152-9

Boakye, M. A. D., Rijal, N. P., Adhikari, U., and Bhattarai, N. (2015). Fabrication and characterization of electrospun PCL-MgO-Keratin-based composite nanofibers for biomedical applications. Materials 8, 4080-4095. doi: $10.3390 / \mathrm{ma} 8074080$

Broughton, G., Janis, J. E., and Attinger, C. E. (2006). Wound healing: an overview. Plast. Reconstr. Surg. 117(Suppl. 7), 1e-S-32e-S. doi: 10.1097/01.prs.0000222562.60260.f9

Cai, Z. X., Mo, X. M., Zhang, K. H., Fan, L. P., Yin, A. L., He, C. L., et al. (2010). Fabrication of chitosan/silk fibroin composite nanofibers for Wound-dressing applications. Int. J. Mol. Sci. 11, 3529-3539. doi: 10.3390/ijms11093529

Cairney, J. W. (2005). Basidiomycete mycelia in forest soils: dimensions, dynamics and roles in nutrient distribution. Mycol. Res. 109, 7-20. doi: 10.1017/S0953756204001753

Campo, G. M., Avenoso, A., Campo, S., Ascola, A. D., Nastasi, G., and Calatroni, A. (2010). Biochimie molecular size hyaluronan differently modulates toll-like receptor-4 in LPS-induced inflammation in mouse chondrocytes. Biochimie 92, 204-215. doi: 10.1016/j.biochi.2009.10.006

Chattopadhyay, S., and Raines, R. T. (2014). Collagen-based biomaterials for wound healing. Biopolymers 101,821-833. doi: 10.1002/bip.22486

Chen, J., Liu, Z., Chen, M., Zhang, H., and Li, X. (2016). Electrospun gelatin fibers with a multiple release of antibiotics accelerate dermal regeneration in infected deep burns. Macromol. Biosci. 16, 1368-1380. doi: 10.1002/mabi.201600108

Chen, R. N., Lee, L. W., Chen, L. C., Ho, H. O., Lui, S. C., Sheu, M. T., et al. (2012). Wound-healing effect of micronized sacchachitin (mSC) nanogel 
on corneal epithelium. Int. J. Nanomed. 7, 4697-4706. doi: 10.2147/IJN.S 34530

Chiu, C. T., Lee, J. S., Chu, C. S., Chang, Y. P., and Wang, Y. J. (2008). Development of two alginate-based wound dressings. J. Mater. Sci. Mater. Med. 19, 2503-2513. doi: 10.1007/s10856-008-3389-2

Choi, J., Panthi, G., Liu, Y., Kim, J., Chae, S., Lee, C., et al. (2015). Keratin/poly (vinyl alcohol) blended nanofibers with high optical transmittance. Polymer 58, 146-152. doi: 10.1016/j.polymer.2014.12.052

Chouhan, D., Chakraborty, B., Nandi, S. K., and Mandal, B. B. (2017). Role of non-mulberry silk fibroin in deposition and regulation of extracellular matrix towards accelerated wound healing. Acta Biomater. 48, 157-174. doi: 10.1016/j.actbio.2016.10.019

Chutipakdeevong, J., Ruktanonchai, U. R., and Supaphol, P. (2013). Process optimization of electrospun silk fibroin fiber mat for accelerated wound healing. J. Appl. Polym. Sci. 3634-3644. doi: 10.1002/app.39611

Croisier, F., and Jérôme, C. (2013). Chitosan-based biomaterials for tissue engineering. Eur. Polym. J. 49, 780-792. doi: 10.1016/j.eurpolymj.2012.12.009

Cui, L., Gong, J., Fan, X., Wang, P., Wang, Q., and Qui, Y. (2013). Transglutaminase-modified wool keratin film and its potential application in tissue engineering. Eng. Life Sci. 13, 149-155. doi: 10.1002/elsc.201100206

Da Silva, M. C. S., Naozuka, J., Maria, J., Luz, R., de Assunção, L. S., Oliveira, P. V., et al. (2012). Enrichment of Pleurotus ostreatus mushrooms with selenium in coffee husks. Food Chem. 131, 558-563. doi: 10.1016/j.foodchem.2011.09.023

Das, S., and Baker, A. B. (2016). Biomaterials and nanotherapeutics for enhancing skin wound healing. Front. Bioeng. Biotechnol. 4:82. doi: 10.3389/fbioe.2016.00082

Dash, M., Chiellini, F., Ottenbrite, R. M., and Chiellini, E. (2011). Chitosan - A versatile semi-synthetic polymer in biomedical applications. Prog. Polymer Sci. 36, 981-1014. doi: 10.1016/j.progpolymsci.2011.02.001

Dhivya, S., Padma, V. V., and Santhini, E. (2015). Wound dressings - a review. BioMedicine 5, 24-28. doi: 10.7603/s40681-015-0022-9

Dogan, G., Başal, G., Bayraktar, O., Özyildiz, F., Uzel, A., and Erdogan, I. (2016). Bioactive sheath/core nanofibers containing olive leaf extract. Microsc. Res. Tech. 79, 38-49. doi: 10.1002/jemt.22603

Dowling, L. S., Crewther, W. G., and Parry, D. A. D. (1986). Secondary structure of component 8c-1 of a-keratin. Biochem. J. 236, 705-712. doi: 10.1042/bj2360705

Dreifke, M. B., Jayasuriya, A. A., and Jayasuriya, A. C. (2015). Current wound healing procedures and potential care. Mater. Sci. Eng. C. 48, 651-662. doi: 10.1016/j.msec.2014.12.068

Edwards, A., Jarvis, D., Hopkins, T., Pixley, S., and Bhattarai, N. (2015). Poly(e-caprolactone)/keratin-based composite nanofibers for biomedical applications. J. Biomed. Mater. Res.Part B Appl. Biomater. 103B, 21-30. doi: $10.1002 /$ jbm.b.33172

Fan, J., Lei, T., Li, J., Zhai, P., Wang, Y., Cao, F., et al. (2016). High protein content keratin/poly (ethylene oxide) nanofibers crosslinked in oxygen atmosphere and its cell culture. JMADE 104, 60-67. doi: 10.1016/j.matdes.2016.05.022

Fan, L., Wang, H., Zhang, K., Cai, Z., and He, C. (2012). Vitamin C-reinforcing silk fibroin nanofibrous matrices for skin care application. RSC Adv. 2, 4110-4119. doi: $10.1039 /$ c2ra20302b

Fraser, R. D. B., Macrae, T. P., and Parry, D. A. D. (1986). Intermediate filaments in a-keratins. Proc. Natl. Acad. Sci. U.S.A. 83, 1179-1183.

Frimpong-Manso, J., Obodai, M., Dzomeku, M., and Apertorgbor, M. M. (2011). Influence of rice husk on biological efficiency and nutrient content of Pleurotus ostreatus (Jacq. ex. Fr.) Kummer. Int. Food Res. J. 18, 249-254.

Fujii, T., and Ide, Y. (2004). Preparation of translucent and flexible human hair protein films and their properties. Biol. Pharm. Bull. 27, 1433-1436. doi: 10.1248/bpb.27.1433

Ghazi, K., Deng-Pichon, U., Warnet, J. M., and Rat, P. (2012). Hyaluronan fragments improve wound healing on in vitro cutaneous model through P2X7 purinoreceptor basal activation: role of molecular weight. PLoS ONE 7:e48351. doi: 10.1371/journal.pone.0048351

Ghica, M. V., Albu Kaya, M. G., Dinu-Pîrvu, C. E., Lupuleasa, D., Udeanu, D. I. (2017). Development, optimization and in vitro/in vivo characterization of collagen-dextran spongious wound dressings loaded with flufenamic acid. Molecules 22:E1552. doi: 10.3390/molecules22091552

Golser, A. V., Ro, M., Bo, H. G., and Scheibel, T. (2018). Engineered collagen: a redox switchable framework for tunable assembly and fabrication of biocompatible surfaces. ACS Biomater. Sci. Eng. 4, 2106-2114. doi: 10.1021/acsbiomaterials.7b00583

Guo, R., Lan, Y., Xue, W., Cheng, B., Zhang, Y., and Wang, C. (2017). Collagencellulose nanocrystal scaffolds containing curcumin-loaded microspheres on infected full-thickness burns repair. J. Tissue Eng. Regen. Med. 11, 3544-3555. doi: 10.1002/term.2272

Guo, S., and Dipietro, L. A. (2010). Factors affecting wound healing. J. Dent. Res. 89, 219-229. doi: 10.1177/0022034509359125

Gurtner, G. C., Werner, S., Barrandon, Y., and Longaker, M. (2008). Wound repair and regeneration. Nature 453, 314-321. doi: 10.1159/000339613

Guzman-Puyol, S., Heredia-Guerrero, J. A., Ceseracciu, L., Hajiali, H., Canale, C., Scarpellini, A., et al. (2016). Low-cost and effective fabrication of biocompatible nanofibers from silk and cellulose-rich materials. ACS Biomater. Sci. Eng. 2, 526-534. doi: 10.1021/acsbiomaterials.5b00500

Hago, E., and Li, X. (2013). Interprenetating polymer network hydrogels based on gelatin and PVA by biocompatible approaches: synthesis and characterization. Adv. Mater. Sci. Eng. 2013:328763. doi: 10.1155/2013/328763

Hajiali, H., Summa, M., Russo, D., Armirotti, A., Brunetti, V., Bertorelli, R., et al. (2016). Alginate-lavender nanofibers with antibacterial and anti-inflammatory activity to effectively promote burn healing. J. Mater. Chem. B. 4, 1686-1695. doi: 10.1039/C5TB02174J

Hall Barrientos, I. J., Paladino, E., Szab,ó, P., Brozio, S., Hall, P. J., Oseghale, C. I., et al. (2017). Electrospun collagen-based nanofibres : a sustainable material for improved antibiotic utilisation in tissue engineering applications. Int. J. Pharm. 531, 67-79. doi: 10.1016/j.ijpharm.2017.08.071

Han, F., Dong, Y., Su, Z., Yin, R., Song, A., and Li, S. (2014). Preparation, characteristics and assessment of a novel gelatin - chitosan sponge scaffold as skin tissue engineering material. Int. J. Pharm. 476, 124-133. doi: 10.1016/j.ijpharm.2014.09.036

Haneef, M., Ceseracciu, L., Canale, C., Bayer, I. S., Heredia-Guerrero, J. A., and Athanassiou, A. (2017). Advanced materials from fungal mycelium: fabrication and tuning of physical properties. Sci. Rep. 7:41292. doi: 10.1038/srep 41292

Hang, Y., Zhang, Y., Jin, Y., Shao, H., and Hu, X. (2012). Preparation of regenerated silk fibroin/silk sericin fibers by coaxial electrospinning. Int. J. Biol. Macromol. 51, 980-986. doi: 10.1016/j.ijbiomac.2012.08.010

He, L. Z., Liu, Y., and Yang, D. (2007). Preparation and performance of chitosangelatin sponge-like wound-healing dressing. J. Clin. Rehabil. Tissue Eng. Res. 11, 5252-5256.

He, M., Zhang, B., Dou, Y., Yin, G., and Chen, X. (2017). Feather keratin / poly (vinyl alcohol) composite. RSC Adv. 7, 9854-9861. doi: 10.1039/C6RA25009B

Hill, P., Brantley, H., and Van Dyke, M. (2010). Some properties of keratin biomaterials: kerateines. Biomaterials 31, 585-593. doi: 10.1016/j.biomaterials.2009.09.076

Hofmann, S., Foo, C. T., Rossetti, F., Textor, M., Vunjak-Novakovic, G., Kaplan, D. L. et al. (2006). Silk fibroin as an organic polymer for controlled drug delivery. J. Control. Release 111, 219-227. doi: 10.1016/j.jconrel.2005.12.009

Hsu, F. Y., Hung, Y. S., Liou, H. M., and Shen, C. H. (2010). Electrospun hyaluronate-collagen nanofibrous matrix and the effects of varying the concentration of hyaluronate on the characteristics of foreskin fibroblast cells. Acta Biomater. 6, 2140-2147 doi: 10.1016/j.actbio.2009.12.023

Hu, X., Shmelev, K., Sun, L., Gil, E. S., Park, S. H., Cebe, P., et al. (2011). Regulation of silk material structure by temperature-controlled water vapor annealing. Biomacromolecules 12, 1686-1696. doi: 10.1021/bm200062a

Huang, S., and Fu, X. (2010). Naturally derived materials-based cell and drug delivery systems in skin regeneration. J. Control. Release 142, 149-159. doi: 10.1016/j.jconrel.2009.10.018

Hung, W. S., Fang, C. L., Su, C. H., Lai, W. F., Chang, Y. C., and Tsai, Y. H. (2001). Cytotoxicity and immunogenicity of SACCHACHITIN and its mechanism of action on skin wound healing. J. Biomed. Mater. Res. 56, 93-100. doi: 10.1002/1097-4636(200107)56:1\&lt;93::AID-JBM1072\&gt;3.0.CO;2-B

Hwang, J. S., Kwon, H. K., Kim, J. E., Rho, J., and Im, S. H. (2012). Immunomodulatory effect of water soluble extract separated from mycelium of Phellinus linteus on experimental atopic dermatitis Immunomodulatory effect of water soluble extract separated from mycelium of Phellinus linteus on experimental atopic dermatitis. BMC Complement. Altern. Med. 12:159. doi: $10.1186 / 1472-6882-12-159$ 
Jaipan, P., Nguyen, A., and Narayan, R. J. (2017). Gelatin-based hydrogels for biomedical applications. MRS Commun. 7, 416-426. doi: 10.1557/mrc.2017.92

Jameton, A., and Pierce, J. (2001). Environment and health: 8. Sustainable health care and emerging ethical responsibilities. CMAJ 164, 365-369.

Jones, M. P., Jones, M., Huynh, T., Dekiwadia, C., Daver, F., and John, S. (2017). Mycelium composites : a review of engineering characteristics and growth kinetics. J. Bionanosci. 11, 241-257. doi: 10.1166/jbns.2017. 1440

Ju, H. W., Lee, O. J., Lee, J. M., Moon, B. M., Park, H. J., Park, Y. R., et al. (2016). Wound healing effect of electrospun silk fibroin nanomatrix in burn-model. Int. J. Biol. Macromol. 85, 29-39. doi: 10.1016/j.ijbiomac.2015.12.055

Karri, V. V. S., Kuppusamy, G., Talluri, S. V., Mannemala, S. S., Kollipara, R., Wadhwani, A. D., et al. (2016). International journal of biological macromolecules curcumin loaded chitosan nanoparticles impregnated into collagen-alginate scaffolds for diabetic wound healing. Int. J. Biol. Macromol. 93, 1519-1529. doi: 10.1016/j.ijbiomac.2016.05.038

Khan, F., and Ahmad, S. R. (2013). Polysaccharides and their derivatives for versatile tissue engineering application. Macromol. Biosci. 13, 395-421. doi: 10.1002/mabi.201200409

Kim, S. Y., Go, K. C., Song, Y. S., Jeong, Y. S., Kim, E. J., and Kim, B. J. (2014). Extract of the mycelium of T. matsutake inhibits elastase activity and TPA-induced MMP-1 expression in human fibroblasts. Int. J. Mol. Med. 34, 1613-1621. doi: 10.3892/ijmm.2014.1969

Koide, T. (2007). Designed triple-helical peptides as tools for collagen biochemistry and matrix engineering. Phil. Trans. R. Soc. B 362, 1281-1291. doi: 10.1098/rstb.2007.2115

Krull, R., Wucherpfennig, T., Eslahpazir, M. E., Walisko, R., Melzer, G., Hempel, D. C., et al. (2013). Characterization and control of fungal morphology for improved production performance in biotechnology. J. Biotechnol. 163, 112-123. doi: 10.1016/j.jbiotec.2012.06.024

Larsen, J. (2015). Biotic interactions in the rhizosphere in relation to plant and soil nutrient dynamics. J. Soil Sci. Plant Nutr. 15, 449-463. doi: $10.4067 /$ S0718-95162015005000039

Li, Y., Wang, Y., Ye, J., Yuan, J., and Xiao, Y. (2016). Fabrication of poly ( $\varepsilon$-caprolactone)/keratin nano fibrous mats as a potential scaffold for vascular tissue engineering. Mater. Sci. Eng. C. 68, 177-183. doi: $10.1016 /$ j.msec.2016.05.117

Liakos, I., Rizzello, L., Bayer, I. S., Pompa, P. P., Cingolani, R., and Athanassiou, A. (2013). Controlled antiseptic release by alginate polymer films and beads. Carbohydr. Polym. 92, 176- 183. doi: 10.1016/j.carbpol.2012.09.034

Liakos, I., Rizzello, L., Hajiali, H., Brunetti, V., Carzino, R., and Pompa, P. P. (2015). Fibrous wound dressings encapsulating essential oils as natural antimicrobial agents. J. Mater. Chem. B Mater. Biol. Med. 3, 1583-1589. doi: $10.1039 /$ С4TB01974A

Liakos, I. L., D'autilia, F., Garzoni, A., Bonferoni, C., Scarpellini, A., Brunetti, V., et al. (2016). All natural cellulose acetate-Lemongrass essential oil antimicrobial nanocapsules. Int. J. Pharm. 510, 508-515. doi: 10.1016/j.ijpharm.2016.01.060

Lian, Y., Zhan, J. C., Zhang, K. H., and Mo, X. M. (2014). Fabrication and characterization of curcumin-loaded silk fibroin/P(LLA-CL) nanofibrous scaffold. Front. Mater. Sci. 8, 354-362. doi: 10.1007/s11706-014-0270-8

Lima, A. C., Mano, J. F., Concheiro, A., and Alvarez-lorenzo, C. (2015). Fast and mild strategy, using superhydrophobic surfaces, to produce collagen / platelet lysate gel beads for skin regeneration. Stem Cell Rev. Rep. 11, 161-179. doi: 10.1007/s12015-014-9548-6

Lin, S., Chen, M., Jiang, H., Fan, L., Sun, B., Yu, F., et al. (2016). Colloids and surfaces B : biointerfaces green electrospun grape seed extractloaded silk fibroin nanofibrous mats with excellent cytocompatibility and antioxidant effect. Colloids Surf. B Biointerf. 139, 156-163. doi: 10.1016/j.colsurfb.2015.12.001

Ma, H., Shen, J., Cao, J., Wang, D., Yue, B., Mao, Z., et al. (2017). Fabrication of wool keratin/polyethylene oxide nano-membrane from wool fabric waste. J. Clean. Prod. 161, 357-361. doi: 10.1016/j.jclepro.2017.05.121

Maeda, N., Miao, J., Simmons, T. J., Dordick, J. S., and Linhardt, R. J. (2014). Composite polysaccharide fibers prepared by electrospinning and coating. Carbohydr. Polym. 102, 950- 955. doi: 10.1016/j.carbpol.2013. 10.038
Martin, P. (1997). Wound healing - aiming for perfect skin regeneration. Science 276, 75-81. doi: $10.1126 /$ science.276.5309.75

Maver, T., Hribernik, S., Mohan, T., Smrke, D. M., Maver, U., and StanaKleinschek, K. (2015). Functional wound dressing materials with highly tunable drug release properties. RSC Adv. 5, 77873-77884. doi: 10.1039/C5RA11972C

Mele, E. (2016). Electrospinning of natural polymers for advanced wound care: towards responsive and adaptive dressings. J. Mater. Chem. B 4, 4801-4812. doi: 10.1039/C6TB00804F

Min, B. M., Jeong, L., Lee, K. Y., and Park, W. H. (2006). Regenerated silk fibroin nanofibers: water vapor-induced structural changes and their effects on the behavior of normal human cells. Macromol. Biosci. 6, 285-292. doi: 10.1002/mabi.200500246

Mogoşanu, G. D., and Grumezescu, A. M. (2014). Natural and synthetic polymers for wounds and burns dressing. Int. J. Pharm. 463, 127- 136 . doi: 10.1016/j.ijpharm.2013.12.015

Moura, L. I., Dias, A. M., Carvalho, E., and De Sousa, H. C. (2013). Recent advances on the development of wound dressings for diabetic foot ulcer treatment: a review. Acta Biomater. 9, 7093-7114. doi: 10.1016/j.actbio.2013.03.033

Nanbu, T., Matsuta, T., Sakagami, H., Shimada, J., Maki, J., and Makino, T. (2011). Anti-UV activity of Lentinus edodes mycelia extract (LEM). In vivo 25, 733-740.

Neel, E. A. A., Bozec, L., Knowles, J. C., Syed, O., Mudera, V., Day, R., et al. (2013). Collagen - emerging collagen based therapies hit the patient. Adv. Drug Deliv. Rev. 65, 429-456. doi: 10.1016/j.addr.2012.08.010

Niyas Ahamed, M. I., and Sastry, T. P. (2011). Wound dressing application of chitosan basedbioactive compounds. Int. J. Pharm. Life Sci. 2, 991-996.

Norouzi, M., Boroujeni, S. M., Omidvarkordshouli, N., and Soleimani, M. (2015). Advances in skin regeneration: application of electrospun scaffolds. Adv. Healthc. Mater. 4, 1114-1133. doi: 10.1002/adhm.201500001

Olsen, D., Yang, C., Bodo, M., Chang, R., Leigh, S., Baez, J., et al. (2003). Recombinant collagen and gelatin for drug delivery. Adv. Drug Deliv. Rev. 55, 1547-1567. doi: 10.1016/j.addr.2003.08.008

Ozaki, Y., Takagi, Y., Mori, H., and Hara, M. (2014). Porous hydrogel of wool keratin prepared by a novel method : an extraction with guanidine / 2mercaptoethanol solution followed by a dialysis. Mater. Sci. Eng. C. 42, 146-154. doi: 10.1016/j.msec.2014.05.018

Parenteau-Bareil, R., Gauvin, R., and Berthod, F. (2010). Collagen-based biomaterials for tissue engineering applications. Materials 3, 1863-1887. doi: $10.3390 / \mathrm{ma} 3031863$

Patel, G., Patel, G., Patel, R., Patel, J., Bharadia, P., and Patel, M. (2007). Sodium alginate: physiological activity, usage \& potential applications. Drug Deliv. Technol. 7, 28-37.

Patrucco, A., Cristofaro, F., Simionati, M., Zoccola, M., Bruni, G., Fassina, L., et al. (2016). Wool fibril sponges with perspective biomedical applications. Mater. Sci. Eng. C., 61, 42-50. doi: 10.1016/j.msec.2015.11.073

Pereira, R. F., Barrias, C. C., Granja, P. L., and Bartolo, P. J. (2013). Advanced biofabrication strategies for skin regeneration and repair. Nanomedicine 8, 603-621. doi: $10.2217 / \mathrm{nnm} .13 .50$

Pignatelli, C., Perotto, G., Nardini, M., Cancedda, R., Mastrogiacomo, M., and Athanassiou, A. (2018). Electrospun silk fibroin fibers for storage and controlled release of human platelet lysate. Acta Biomater. 73, 365-376. doi: 10.1016/j.actbio.2018.04.025

Pinkas, D. M., Ding, S., Raines, R. T., and Barron, A. E. (2011). Tunable, posttranslational hydroxylation of collagen domains in Escherichia coli. ACS Chem. Biol. 6, 320-324. doi: 10.1021/cb100298r

Powell, H. M., Supp, D. M., and Boyce, S. T. (2008). Influence of electrospun collagen on wound contraction of engineered skin substitutes. Biomaterials 29, 834-843. doi: 10.1016/j.biomaterials.2007.10.036

Pritchard, E. M., Valentin, T., Panilaitis, B., Omenetto, F., and Kaplan, D. L. (2013). Antibiotic-releasing silk biomaterials for infection prevention and treatment. Adv. Funct. Mater. 23, 854-861. doi: 10.1002/adfm.201201636

Qu, Y., Cao, C., Wu, Q., Huang, A., Song, Y., Li, H. (2018). The dual delivery of KGF and bFGF by collagen membrane to promote skin wound healing. J. Tissue Eng. Regen. Med. 12, 1508-1518. doi: 10.1002/term.2691

Reichl, S. (2009). Biomaterials films based on human hair keratin as substrates for cell culture and tissue engineering. Biomaterials 30, 6854-6866. doi: 10.1016/j.biomaterials.2009.08.051 
Reimers, K., Liebsch, C., Radtke, C., Kuhbier, J. W., and Vogt, P. M. (2015). Silks as scaffolds for skin reconstruction. Biotechnol. Bioeng. 112, 2201-2205. doi: 10.1002/bit.25654

Richter, J. R., De Guzman, R. C., Greengauz-Roberts, O. K., and Van Dyke, M. (2012). Structure-property relationships of meta-kerateine biomaterials derived from human hair. Acta Biomater. 8, 274-281. doi: 10.1016/j.actbio.2011. 08.020

Rieger, K. A., Birch, N. P., and Schiffman, J. D. (2013). Designing electrospun nanofiber mats to promote wound healing - a review. J. Mater. Chem., B. 1, 4531-4541. doi: 10.1039/c3tb20795a

Rieger, K. A., and Schiffman, J. D. (2014). Electrospinning an essential oil : cinnamaldehyde enhances the antimicrobial efficacy of chitosan / poly (ethylene oxide) nanofibers. Carbohydr. Polym. 113, 561-568. doi: 10.1016/j.carbpol.2014.06.075

Roh, D. H., Kang, S. Y., Kim, J. Y., Kwon, Y. B.,Young Kweon, H., Lee, K. G., et al. (2006). Wound healing effect of silk fibroin/alginate-blended sponge in full thickness skin defect of rat. J. Mater. Sci. Mater. Med. 17, 547-552. doi: 0.1007/s10856-006-8938-y

Romano, I., Ayadi, F., Rizzello, L., Summa, M., Bertorelli, R., Pompa, P. P., et al. (2015a). Controlled antiseptic/eosin release from chitosan-based hydrogel modified fibrous substrates. Carbohydr. Polym. 92, 176- 183. doi: 10.1016/j.carbpol.2015.05.057

Romano, I., Mele, E., Heredia-Guerrero, J. A., Ceseracciu, L., Hajiali, H., Goldoni, L., et al. (2015b). Photo-polymerisable electrospun fibres of N-methacrylate glycol chitosan for biomedical applications. RSC Adv. 5, 24723-24728. doi: 10.1039/C5RA02301G

Rouse, J. G., and Van Dyke, M. E. (2010). A review of keratin-based biomaterials for biomedical applications. Materials 3, 999-1014. doi: 10.3390/ma3020999

Salati, S., Imporzano, G. D., Menin, B., Veronesi, D., Scaglia, B., Abbruscato, P., et al. (2017). Bioresource technology mixotrophic cultivation of chlorella for local protein production using. Bioresour. Technol. 230, 82-89. doi: 10.1016/j.biortech.2017.01.030

Saul, J. M., Ellenburg, M. D., de Guzman, R. C., and Van Dyke M (2011). Keratin hydrogels support the sustained release of bioactive ciprofloxacin. Biomed Mater Res Part A 98A, 544-553. doi: 10.1002/jbm.a.33147

Schneider, A., Wang, X. Y., Kaplan, D. L., Garlick, J. A., and Egles, C. (2009). Biofunctionalized electrospun silk mats as a topical bioactive dressing for accelerated wound healing. Acta Biomater. 5, 2570-2578. doi: 10.1016/j.actbio.2008.12.013

Sell, S., Barnes, C., Smith, M., McClure, M., Madurantakam, P., Grant, J., et al. (2007). Extracellular matrix regenerated: tissue engineering via electrospun biomimetic nanofibers. Polym. Int. 56, 1349-1360. doi: 10.1002/pi.2344

Sell, S. A., Wolfe, P. S., Garg, K., McCool, J. M., Rodriguez, I. A., and Bowlin, G. L. (2010). The use of natural polymers in tissue engineering: a focus on electrospun extracellular matrix analogues. Polymers 2, 522-553. doi: $10.3390 /$ polym 2040522

Selvaraj, S., and Fathima, N. N. (2017). Fenugreek incorporated silk fibroin nanofibers - A potential antioxidant scaffold for enhanced wound healing. ACS Appl. Mater. Interf. 9, 5916-5926. doi: 10.1021/acsami.6b16306

Sen, C. K., Gordillo, G. M., Roy, S., Kirsner, R., Lambert, L., Hunt, T. K., et al. (2009). Human skin wounds: a major and snowballing threat to public health and the economy. Wound Repair Regen. 17, 763-771. doi: 10.1111/j.1524-475X.2009.00543.x

Setti, C., Suarato, G., Perotto, G., Athanassiou, A., and Bayer, I. S. (2018). Investigation of in vitro hydrophilic and hydrophobic dual drug release from polymeric films produced by sodium alginate-MaterBi ${ }^{\circledR}$ drying emulsions. Eur. J. Pharm. Biopharm. 130, 71-82. doi: 10.1016/j.ejpb.2018.06.019

Shahverdi, S., Hajimiri, M., Amin, M., Akbar, A., and Dinarvand, R. (2014). Fabrication and structure analysis of poly (lactide-co-glycolic acid)/ silk fibroin hybrid scaffold for wound dressing applications. Int. J. Pharm. 473, 345-355. doi: 10.1016/j.ijpharm.2014.07.021

Shan, Y. H., Peng, L. H., Liu, X., Chen, X., Xiong, J., and Gao, J. Q. (2015). Silk fibroin / gelatin electrospun nanofibrous dressing functionalized with astragaloside IV induces healing and anti-scar effects on burn wound. Int. J. Pharm. 479, 291-301. doi: 10.1016/j.ijpharm.2014.12.067

Shanmugam, K., and Sundaramoorthy, S. (2015). Development and characterization of an electrospun mat from Eri silk fibroin and PLA blends. RSC Adv. 5, 31352-31364. doi: 10.1039/C4RA15268A
Sharma, S., and Gupta, A. (2016). Sustainable management of keratin waste biomass: applications and future perspectives. Braz. Arch. Biol. Technol. Int. J. 59:e16150684. doi: 10.1590/1678-4324-2016150684

Shavandi, A., Silva, T. H., Bekhit, A. A., and Bekhit, A. E. A.(2017). Keratin: dissolution, extraction and biomedical application. Biomater. Sci. 5, 1699-1735. doi: 10.1039/C7BM00411G

Sheng, X., Fan, L., He, C., Zhang, K., and Mo, X. (2013). Vitamin E-loaded silk fibroin nanofibrous mats fabricated by green process for skin care application. Int. J. Biol. Macromol. 56, 49-56. doi: 10.1016/j.ijbiomac.2013. 01.029

Silva, S. S., Santos, T. C., Cerqueira, M. T., Marques, A. P., Reys, L. L., Silva, T. H., et al. (2012). The use of ionic liquids in the processing of chitosan/silk hydrogels for biomedical applications. Green Chem. 14, 1463-1470. doi: 10.1039/c2gc16535j

Singaravelu, S., Ramanathan, G., Muthukumar, T., Raja, M. D., Nagiah, N., Thyagarajan, S., et al. (2016). Durable keratin-based bilayered electrospun mats for wound closure. J. Mater. Chem. B 3982, 3982-3997. doi: 10.1039/C6TB00720A

Sinno, H., and Prakash, S. (2013). Complements and the wound healing cascade : an updated review. Plastic Surg. Int. 2013:146764. doi: 10.1155/2013/146764

Srivastava, C. M., Purwar, R., Kannaujia, R., and Sharma, D. (2015). Flexible silk fibroin films for wound dressing. Fibers Polym. 5, 1020-1030. doi: 10.1007/s12221-015-1020-y

Steinert, P. M., and Marekov, L. N. (1993). Keratin intermediate filament structure. J. Mol. Biol. 230, 436-452. doi: 10.1006/jmbi.1993.1161

Su, C. H., Liu, S. H., Yu, S. Y., Hsieh, Y. L., Ho, H. O., Hu, C. H., et al. (2005). Development of fungal mycelia as a skin substitute: characterization of keratinocyte proliferation and matrix metalloproteinase expression during improvement in the wound-healing process. J. Biomed. Mater. Res.Part A 72, 220-227. doi: 10.1002/jbm.a.30235

Su, C. H., Sun, C. S., Juan, S. W., Ho, H. O., Hu, C. H., and Sheu, M. T. (1999). Development of fungal mycelia as skin substitutes: effects on wound healing and fibroblast. Biomaterials 20, 61-68. doi: 10.1016/S0142-9612(98)00139-2

Su, C. H., Sun, C. S., Juan, S. W., Hu, C. H., Ke, W. T., and Sheu, M. T. (1997). Fungal mycelia as the source of chitin and polysaccharides and their applications as skin substitutes. Biomaterials 18, 1169-1174. doi: 10.1016/S0142-9612(97)00048-3

Suganya, S., Venugopal, J., Ramakrishna, S., Lakshmi, B. S., and Dev, V. R. (2014). Naturally derived biofunctional nanofibrous scaffold for skin tissue regeneration. Int. J. Biol. Macromol. 68, 135-143. doi: 10.1016/j.ijbiomac.2014.04.031

Summa, M., Russo, D., Penna, I., Margaroli, N., Bayer, I. S., Bandiera, T., et al. (2018). A biocompatible sodium alginate/povidone iodine film enhances wound healing. Eur. J. Pharm. Biopharm. 122, 17-24. doi: 10.1016/j.ejpb.2017.10.004

Synytsya, A., and Novák, M. (2013). Structural diversity of fungal glucans. Carbohydr. Polym. 92, 792-809. doi: 10.1016/j.carbpol.2012.09.077

Szekalska, M., Puciłowska, A., Szymanska, E., Ciosek, P., and Winnicka, K. (2016). Alginate: current use and future perspectives in pharmaceutical and biomedical applications. Int. J. Polym. Sci. 2016:7697031. doi: 10.1155/2016/7697031

Tachibana, A., Furuta, Y., Takeshima, H., Tanabe, T., and Yamauchi, K. (2002). Fabrication of wool keratin sponge scaffolds for long-term cell cultivation. J. Biotechnol. 93, 165-170. doi: 10.1016/S0168-1656(01)00395-9

Thanusha, A. V., Dinda, A. K., and Koul, V. (2018). Evaluation of nano hydrogel composite based on gelatin/HA/CS suffused with Asiatic acid/ZnO and $\mathrm{CuO}$ nanoparticles for second degree burns. Mater. Sci. Eng. C 89, 378-386. doi: 10.1016/j.msec.2018.03.034

Thyagarajan, S. L., Ramanathan, G., Singaravelu, S., Kandhasamy, S., Perumal, P. T., and Sivagnanam, U. T. (2017). Characterization and evaluation of siderophore-loaded gelatin microspheres : a potent tool for wound-dressing material. Polym. Bull. 74, 2349-2363. doi: 10.1007/s00289-016-1840-y

Tolg, C., Telmer, P., and Turley, E. (2014). Specific sizes of hyaluronan oligosaccharides stimulate fibroblast migration and excisional wound repair. PLoS ONE 9:e88479. doi: 10.1371/journal.pone.0088479

Tonin, C., Aluigi, A., Vineis, C., Varesano, A., Montarsolo, A., and Ferrero, F. (2007). Thermal and structural characterization of Poly(ethyleneoxide)/Keratin blend films. J. Therm. Anal. Calorim. 89, 601-608. doi: $10.1007 /$ s10973-006-7557-7 
Uppal, R., Ramaswamy, G. N., Arnold, C., Goodband, R., and Wang, Y. (2011). Hyaluronic acid nanofiber wound dressing-production, characterization, and in vivo behavior. J. Biomed. Mater. Res.Part B Appl. Biomater. 97B, 20-29. doi: 10.1002/jbm.b.31776

Vamanu, E. (2014). Antioxidant properties of mushroom mycelia obtained by batch cultivation and tocopherol content affected by extraction procedures. Biomed Res. Int. 2014:974804. doi: 10.1155/2014/974804

Vassilev, N., Baca, M., Vassileva, M., Franco, I., and Azcon, R. (1995). Rock phosphate solubilization by Aspergillus niger grown on sugar-beet waste medium. Appl. Microbiol. Biotechnol. 44, 546-549. doi: 10.1007/BF001 69958

Veleirinho, B., Coelho, D. S., Dias, P. F., Maraschin, M., Ribeiro-do-valle, R. M., and Lopes-da-silva, J. A. (2012). Nanofibrous pol7(3-hydroxybutyrateco-3-hydroxyvalerate)/chitosan scaffolds for skin regeneration. Int. J. Biol. Macromol. 51, 343-350. doi: 10.1016/j.ijbiomac.2012.05.023

Verma, V., Verma, P., Ray, P., and Ray, A. R. (2008). Preparation of scaffolds from human hair proteins for tissue-engineering applications. Biomed. Mater. 3:025007. doi: 10.1088/1748-6041/3/2/025007

Vyas, K. S., and Vasconez, H. C. (2014). Wound healing: biologics, skin substitutes, biomembranes and scaffolds. Healthcare 2, 356-400. doi: 10.3390/healthcare2030356

Wang, B., Yang, W., McKittrick, J., and Meyers, M. A. (2016). Keratin: structure, mechanical properties, occurrence in biological organisms, and efforts at bioinspiration. Prog. Mater. Sci. 76, 229-318. doi: 10.1016/j.pmatsci.2015.06.001

Wang, J., Hao, S., Luo, T., Zhou, T., Yang, X., and Wang, B. (2017). Keratose / poly (vinyl alcohol) blended nanofibers : fabrication and biocompatibility assessment. Mater. Sci. Eng C 72, 212-219. doi: 10.1016/j.msec.2016. 11.071

Wang, S., Taraballi, F., Tan, L. P., and Ng, K. W. (2012). Human keratin hydrogels support fibroblast attachment and proliferation in vitro. Cell Tissue Res. 347, 795-802. doi: 10.1007/s00441-011-1295-2

Wani, M. Y., Hasan, N., and Malik, M. A. (2010). Chitosan and Aloe vera: two gifts of nature. J. Dispers. Sci. Technol. 31, 799-811. doi: 10.1080/01932690903333606

Wei, G., and Ma, P. X. (2008). Nanostructured biomaterials for regeneration. Adv. Funct. Mater. 18, 3568-3582. doi: 10.1002/adfm.200800662

Wei, Y., Chang, Y. H., Liu, C. J., and Chung, R. J. (2018). Integrated Oxidized-Hyaluronic Acid/Collagen Hydrogel with $\beta$-TCP using Proanthocyanidins as a crosslinker for drug delivery. Pharmaceutics 10:E37. doi: 10.3390/pharmaceutics10020037

Weller, C., and Sussman, G. (2006). Wound dressings update. J. Pharm. Prac. Res. 36, 318-324. doi: 10.1002/j.2055-2335.2006.tb00640.x

Wharram, S. E., Zhang, X., Kaplan, D. L., and McCarthy, S. P. (2010). Electrospun silk material systems for wound healing. Macromol. Biosci. 10, 246-257. doi: 10.1002/mabi.200900274

Xu, S., Li, J., He, A., Liu, W., Jiang, X., Zheng, J., et al. (2009). Chemical crosslinking and biophysical properties of electrospun hyaluronic acid based ultra-thin fibrous membranes. Polymer 50, 3762-3769. doi: 10.1016/j.polymer.2009.06.009

Xu, S., Sang, L., Zhang, Y., Wang, X., and Li, X. (2013). Biological evaluation of human hair keratin scaffolds for skin wound repair and regeneration. Mater. Sci. Eng C 33, 648-655. doi: 10.1016/j.msec.2012.10.011

Yamauchi, K., Hojo, H., Yamamoto, Y., and Tanabe, T. (2003). Enhanced cell adhesion on RGDS-carrying keratin film. Mater. Sci. Eng. C 23, 467-472. doi: 10.1016/S0928-4931(02)00280-1
Yan, J., Wang, W., and Wu, J. (2014). Recent advances in Cordyceps sinensis polysaccharides : mycelial fermentation, isolation, structure, and bioactivities : a review. J. Funct. Foods 6, 33-47. doi: 10.1016/j.jff.2013.11.024

Yan, S., Zhang, Q., Wang, J., Liu, Y., Lu, S., Li, M., et al. (2013). Silk fibroin/chondroitin sulfate/hyaluronic acid ternary scaffolds for dermal tissue reconstruction. Acta Biomater. 9, 6771-6782. doi: 10.1016/j.actbio.2013.02.016

Yang, X., Fan, L., Ma, L., Wang, Y., Lin, S., Yu, F., et al. (2017). Green electrospun Manuka honey/silk fibroin fibrous matrices as potential wound dressing. Mater. Design 119, 76-84. doi: 10.1016/j.matdes.2017.01.023

Yao, C. H., Lee, C. Y., Huang, C. H., Chen, Y. S., and Chen, K. Y. (2017). Novel bilayer wound dressing based on electrospun gelatin/keratin nanofibrous mats for skin wound repair. Mater. Sci. Eng. C 79, 533-540. doi: 10.1016/j.msec.2017.05.076

Yen, K.-C., Chen, C.-Y., Huang, J.-Y., Kuo, W.-T., and Lin, F.-H. (2016). Fabrication of keratin/fibroin membranes by electrospinning for vascular tissue engineering. J. Mater. Chem. B 4, 237-244. doi: 10.1039/C5TB01921D

Yoon, D., Yoon, D., Cha, H., and Lee, J. (2018). Enhancement of wound healing efficiency mediated by artificial dermis functionalized with EGF or NRG1 Enhancement of wound healing efficiency mediated by artificial dermis functionalized with EGF or NRG1. Biomed. Mater. 13:045007. doi: 10.1088/1748-605X/aaac37

Yuan, J., Geng, J., Xing, Z., Shim, K. J., Han, I., and Kim, J. C. (2015). Novel wound dressing based on nanofibrous PHBV - keratin mats. J. Tissue Eng. Regen. Med. 9, 1027-1035. doi: 10.1002/term.1653

Zhao, W., Liu, W., Li, J., Lin, X., and Wang, Y. (2015). Preparation of animal polysaccharides nanofibers by electrospinning and their potential biomedical applications. J. Biomed. Mater. Res.Part A 103A, 807-818. doi: 10.1002/jbm.a.35187

Zhong, S. P., Zhang, Y. Z., and Lim, C. T. (2010). Tissue scaffolds for skin wound healing and dermal reconstruction. Wiley Interdiscip. Rev. Nanomed. Nanobiotechnol. 2, 510-525. doi: 10.1002/wnan.100

Zhu, C., Lei, H., Fan, D., and Duan, Z. (2018). Novel enzymatic crosslinked hydrogels that mimic extracellular matrix for skin wound healing. J. Mater. Sci. 53, 5909-5928. doi: 10.1007/s10853-017-1956-y

Zhu, H., Li, R., Wu, X., Chen, K., and Che, J. (2017). Controllable fabrication and characterization of hydrophilic PCL/wool keratin nanonets by electronetting. Eur. Polym. J. 86, 154-161. doi: 10.1016/j.eurpolymj.2016.11.023

Zhu, J., Huang, W., Zhang, Q., Ling, S., Chen, Y., and Kaplan, D. L. (2016). Aqueous-based coaxial electrospinning of genetically engineered silk elastin core-shell nanofibers. Materials 9:E221. doi: 10.3390/ma9040221

Zoccola, M., Aluigi, A., Vineis, C., Tonin, C., Ferrero, F., and Piacentino, M. G. (2008). Study on cast membranes and electrospun nanofibers made from keratin/fibroin blends. Biomacromolecules 9, 2819-2825. doi: $10.1021 / \mathrm{bm} 800579 \mathrm{a}$

Conflict of Interest Statement: The authors declare that the research was conducted in the absence of any commercial or financial relationships that could be construed as a potential conflict of interest.

Copyright (C) 2018 Suarato, Bertorelli and Athanassiou. This is an open-access article distributed under the terms of the Creative Commons Attribution License (CC BY). The use, distribution or reproduction in other forums is permitted, provided the original author(s) and the copyright owner(s) are credited and that the original publication in this journal is cited, in accordance with accepted academic practice. No use, distribution or reproduction is permitted which does not comply with these terms. 